\title{
Adverse Drug Reactions and associated factors among adult HIV-positive patients taking ART at the Yaoundé Central Hospital, Cameroon
}

Carine Nguefeu Nkenfou-Tchinda ${ }^{1 *}$, Barbara Atogho Tiedeu ${ }^{1}$, Celine Nguefeu Nkenfou ${ }^{2}$, Akindeh M. Nji ${ }^{1}$, Aristid Ekollo Mbange $^{1}$, Jean Paul Kengne Chedjou ${ }^{1}$, Calvino Tah Fomboh' ${ }^{3}$, Charles Kouanfack ${ }^{4}$, Wilfred F. Mbacham ${ }^{1}$

${ }^{1}$ Department of Biochemistry, Faculty of Science, University of Yaound I, Yaoundé, Cameroon.

${ }^{2}$ Systems Biology Laboratory, Chantal Biya International Reference Centre for Research on HIV and AIDS Prevention and Management, Yaound, Cameroon. ${ }^{3}$ The Biotechnology Center, University of Yaound I, Yaoundé, Cameroon.

${ }^{4}$ Department of Public Health, Faculty of Medicine and Pharmaceutical Sciences, University of Dschang, Dschang, Cameroon.

\section{ARTICLE INFO \\ Received on: $18 / 06 / 2020$ \\ Accepted on: 14/09/2020 \\ Available online: 05/11/2020}

\section{Key words:}

Adverse drug reactions, associated factors, ART.

\begin{abstract}
Highly Active Anti-Retroviral Therapy has considerably enhanced the life span and quality of life of people living with Human Immunodeficiency Virus (HIV)/Acquired Immune Deficiency Syndrome (AIDS). Unfortunately, these drugs have been associated with some Adverse Drug Reactions (ADRs). This study assessed the general profile and prevalence of ADRs and their associated factors at the Yaoundé Central Hospital in Cameroon. Data were obtained from patients clinical records. Statistical analyses were done using the Statistical Package for the Social Sciences software version 25. The medical files of 1,254 HIV/AIDS patients who initiated antiretroviral therapy (ART) were included in this study, 306 (24.40\%) of whom had reported to have developed at least one ADR. The most common biological systems affected were the hematological, systemic, gastro-intestinal, dermatological and central nervous system with $37.58 \%, 12.75 \%, 12.75 \%, 12.75 \%$ and $10.78 \%$ respectively. Factors that were significantly associated with the development of ADRs included age $(p=0.003)$, CD4 cell count $(p=0.004)$, Cotrimoxazole prophylaxis $(p=$ $0.029)$ and ART regimen $(p<0.001)$. Additionally we found that about $88.56 \%$ of patients who developed ADR were within their first 3 months of treatment. It is important to put in place a good reporting system for the early detection and prevention of ADRs.
\end{abstract}

\section{BACKGROUND}

Acquired Immune Deficiency Syndrome (AIDS) is a disease caused by the Human Immunodeficiency Virus (HIV). The World Health Organization (WHO) recently reported an estimated 37.9 million people infected worldwide in 2018. Africa is seriously affected, accounting for approximately $70 \%$ of all the people infected around the world (UNAIDS/WHO, 2019). Cameroon is not excluded from this picture, with an estimated average prevalence of $3.8 \%$ among the young adult population aged between 15 and 49 years (UNAIDS/WHO/UNICEF, 2017). Up until now, no effective

\section{"Corresponding Author}

Carine Nguefeu Nkenfou-Tchinda, Department of Biochemistry, Faculty of Science, University of Yaound I, Yaoundé, Cameroon.

E-mail:nguefeucarine@yahoo.fr cure or vaccine against HIV/AIDS exists; nevertheless, it is controlled by the administration of Antiretroviral Therapy (ART).

Since its introduction, the therapy has considerably reduced the morbidity and mortality caused by HIV infection (Anlay et al., 2016). Anti-HIV drugs act by preventing viral multiplication, thereby improving the immune system's response and decreasing the risk of transmission to sexual partners and children (Cohen et al., 2016). Unfortunately, alongside these gains, antiretrovirals, like many other administered drugs, are reported to be associated with Adverse Drug Reactions (ADRs). Anti-HIV-related ADRs have been particularly found to occur in higher proportions at the beginning of ART (Tadesse et al., 2014). An ADR is any noxious, unintended, and undesired effect of a drug, which occurs at normal doses used in humans (Ejigu et al., 2018). The outcome of ADRs is observed in both adults and children (Oumar et al., 2012). 
Many studies have been carried out around the world, and the results from these studies have shown that the incidence of ADRs among patients on ART ranged between $4.3 \%$ and $90 \%$ (Kindie et al., 2017; Mehari et al., 2017; Roshni et al., 2016; Shet et al., 2014). Some factors including the age of the patient, gender, ART regimen, duration of treatment, opportunistic infection prophylaxis, WHO's clinical stage, disease biomarkers, and body mass index were shown to be associated with the development of ADR (Bhatnagar et al., 2013; Ejigu et al., 2018; Kindie et al., 2017; Kumari et al., 2017; Lartey et al., 2014; Masenyetse et al., 2015; Raikar et al., 2018).

The weakness of the immune system due to the infection and the complexity of anti-HIV drugs can also affect the occurrence of ADRs (Hawkins, 2010). Previous studies have described associations between ART use and a large spectrum of ADRs. These included lipodystrophy, abdominal pains, fatigue, nausea and vomiting, diarrhea, hepatotoxicity, hypersensitivity syndrome reactions (rashes), Central Nervous System (CNS) adverse events, and pancreas and kidney toxicities (Neuman et al., 2012; Reust, 2011; Shubber et al., 2013). The severity of ADRs ranges from mild to life-threatening and may occur following a single dose or prolonged administration of the medicine. The combination of two or more medicines may also aggravate the condition (Eluwa et al., 2012). Drug toxicities can add to the complexity of the disease's management by affecting patient compliance with the treatment. The plausible resulting consequences could be the poor response to treatment associated with higher costs to the public health system (Fagbami et al., 2015; Hansana et al., 2013; Mehari et al., 2017; Rajesh et al., 2012). Generally, about $6 \%$ of all admissions into medical hospital wards have been shown to be due to ADRs (Ibrahim Elnagar, 2017). Some pieces of evidence have shown that, in up to one-quarter of patients, there was a modification of the initial ART regimen due to ADRs (Kindie et al., 2017; Lima et al., 2012).

It is important to note that the chosen ART regimen should not only maintain viral suppression but also ensure that this regimen is safe so that the patient complies with the therapy. Very few studies have been conducted on adverse events associated with the therapy and the predicting factors in ART programs across Cameroon. This study was hence carried out to gain knowledge on the profile of ADR associated with Anti-Retroviral (ARV) drugs and their prevalence in the Central Hospital of Yaounde. Factors associated with ADRs were also investigated, with the overall goal of improving the management of patients harboring the HIV infection.

\section{MATERIALS AND METHODS}

\section{Study design and setting}

A retrospective study was conducted in the outpatient ART Center (Day Care Hospital) of the Central Hospital of Yaounde, the second largest city and political capital of Cameroon. This center is one of the largest centers in the country, with approximately $200 \mathrm{HIV-positive} \mathrm{individuals} \mathrm{enrolling} \mathrm{themselves}$ in the program each month. The data for this study were obtained by reviewing the medical records of patients who had been enrolled in the center between 1st January 2013 and 31st December 2013.

\section{Study population}

The study population included all HIV-positive patients recruited into or enrolled in the program at the ART Center between 1 January 2013 and 31 December 2013 and who had had at least one follow-up clinical visit after commencing treatment. According to the standard procedure at the center during that period, once the HIV status was confirmed to be positive, medical information was obtained by health workers during the initial visit through a standardized interview (including detailed socioeconomic and demographic data) and entered into the patient's cards. The patient then underwent a clinical consultation by a medical doctor to determine his/her WHO's clinical stage with regard to the condition. After this, a set of baseline laboratory tests (hematology, biochemistry, and CD4+ cell count) was required from the patients. The test results were then analyzed by the physician and the patient was classified as eligible or not eligible for ART initiation. Once declared eligible, the patients were initiated on a combination ART consisting of two nucleos(t)ide reverse transcriptase inhibitors (NRTIs) [zidovudine (AZT)/lamivudine (3TC) or tenofovir (TDF)/3TC) plus a nonNRTI (NNRTI) (either Nevirapine (NVP) or Efavirenz (EFV)]. All patients were generally given a 30-days supply at initiation. Thereafter, they were seen again once more and interviewed. After the first prescription pick-up, a maximum of 3 months treatment at a time was dispensed to the patient depending on his/her tolerance and compliance with the medication. Follow-up tests were done every 6 months, unless otherwise indicated. All patient data and test results were kept in their medical records.

\section{Data collection procedure}

ADRs were identified based on patient complaints and/ or observations made and reported in the patient's record by physicians during the routine clinical examination. Physicians considered that an adverse effect was associated with ART if it was absent prior to its initiation and to which other causes could not be attributed. ADRs were considered as minor if the patient continued with the same medicines or serious if he was given other medications.

Data for this study were retrieved from available patient medical records found in standardized data collection forms. Baseline demographic characteristics (age, marital status, sex, employment status, geographic site of residence, and educational level) and clinical/immunological factors (WHO's clinical stage, ART regimen, hemoglobin level, weight, TB coinfection, cotrimoxazole prophylaxis, poor compliance history, and CD4+ cell count) were collected. The baseline was defined as taken at the time of ART initiation. The onset time of ADRs was evaluated by estimating the time between the treatment initiation date and the date of complaints reported or the date of the test results for laboratory-based ADRs.

\section{Ethical considerations}

Direct patient identifiers, like names, were not collected during this retrospective study; instead unique codes were used. All the data obtained during the course of the study were kept confidential. Ethical committee approval was obtained from the 
National Ethics Committee for Research in Human Health of Cameroon (No. 2014/12/670/CE/CNERSH/SP).

\section{Data analysis}

Data were analyzed using the Statistical Package for the Social Sciences version 25 to relate variables and/or compare groups in terms of variables. Descriptive and univariate analyses were carried out on quantitative data. The association between different factors and ADRs was estimated with a 95\% confidence interval, using the chi-square test. A $p$-value $<0.05$ was considered to be statistically significant. The studied risk factors included sex, age, weight, WHO's stage, CD4 cell count, ART regimen, and administration of cotrimoxazole prophylaxis.

\section{RESULTS}

\section{Sociodemographic characteristics}

A total of 2,108 patients were enrolled in the ART program between January and December 2013. Only 1996 medical files were available and screened, while 112 were missing or unavailable. Among the files reviewed, 645 were excluded which belonged to patients who never initiated the treatment and 97 belonged to patients who were transferred in (who initiated treatment elsewhere and were transferred to the Central Hospital for diverse reasons). This last category was excluded from the analysis because most of the time, the data on their past clinical/ medical history were insufficient. For this study, therefore, we finally included 1,254 patients' records. Table 1 shows the sociodemographic characteristics of the study population. The percentage of females was relatively larger than that of males, $845(67.4 \%)$ versus 409 (32.6\%). The age group of 25 and 34

Table 1. General or socio-demographic characteristics of patients at treatment initiation.

\begin{tabular}{|c|c|c|c|}
\hline Variables & Category & Number & Percentage (\%) \\
\hline \multirow[t]{2}{*}{ Sex } & Female & 845 & 67.4 \\
\hline & Male & 409 & 32.6 \\
\hline \multirow[t]{4}{*}{ Age } & $15-24$ & 90 & 7.2 \\
\hline & $25-34$ & 430 & 34.3 \\
\hline & $35-44$ & 398 & 31.7 \\
\hline & $\geq 45$ & 336 & 26.8 \\
\hline \multirow[t]{5}{*}{ Marital status } & Married & 294 & 23.4 \\
\hline & Cohabiting & 225 & 17.9 \\
\hline & Single & 547 & 43.6 \\
\hline & Widowed & 145 & 11.6 \\
\hline & Divorced & 43 & 3.4 \\
\hline \multirow[t]{3}{*}{ Employment status } & Employed & 361 & 28.8 \\
\hline & Self-employed & 424 & 33.8 \\
\hline & Unemployed & 469 & 37.4 \\
\hline \multirow[t]{2}{*}{ Site of residence } & In Yaoundé & 1106 & 88.2 \\
\hline & Out of Yaoundé & 148 & 11.8 \\
\hline \multirow[t]{4}{*}{ Educational level } & None & 40 & 3.2 \\
\hline & Primary & 438 & 34.9 \\
\hline & Secondary & 654 & 52.2 \\
\hline & Tertiary & 122 & 9.7 \\
\hline
\end{tabular}

years yielded the highest number of patients, with $430(34.3 \%)$ participants. Concerning the level of education, $654(52.2 \%)$ participants had a secondary school education. The number of people who were reported to be single was 547 (43.6\%); 469 individuals (37.4\%) were unemployed; and 1,106 (88.2\%) reported to be living in Yaounde.

\section{Clinical and immunological characteristics}

Out of the 1,254 patients who initiated treatment, more than half $(55.7 \%, 699)$ of them had a bodyweight of more than $60 \mathrm{~kg}$. One-third $(33.2 \%)$ of them were at WHO's stage III at the moment of treatment initiation. Study subjects with a CD $4<200$ cells $/ \mu$ accounted for $57.7 \%$. The number of patients who had received cotrimoxazole prophylaxis was $463(36.9 \%)$ and $7.5 \%$ had TB coinfection. The two predominant ART regimens initially prescribed for the patients during the data collection period were AZT/3TC/NVP (46.6\%, 584), followed by TDF/3TC/EFV (40.9\%, 513). Individuals classified under "Others" for the ART regimen were those who were included in a clinical trial research study that was going on at the Day Care Hospital during that period. One-fifth $(21.9 \%)$ of the patients had a history of self-reported poor compliance (defined here as skipping doses or interrupting the treatment for a short or a long period of time) in their medical files (Table 2).

\section{General profile of ADRs in the study population}

The profile of ADRs in this study is shown in Table 3. A broad variety of specific ADRs was reported and they were classified into eight different biological systems or groups, namely gastrointestinal (GI), dermatological (DMT), CNS, peripheral

Table 2. Clinical and immunological characteristics of the study population.

\begin{tabular}{|c|c|c|c|}
\hline Variables & Category & Number & Percentage $(\%)$ \\
\hline \multirow[t]{3}{*}{ Weight (Kg) } & $<50$ & 122 & 9.7 \\
\hline & $50-60$ & 433 & 34.5 \\
\hline & $>60$ & 699 & 55.7 \\
\hline \multirow[t]{4}{*}{ WHO stage } & I & 407 & 32.5 \\
\hline & II & 351 & 28.0 \\
\hline & III & 416 & 33.2 \\
\hline & IV & 80 & 6.4 \\
\hline \multirow[t]{3}{*}{ CD4 cell count $($ cells $/ \mu 1)$} & $\leq 200$ & 724 & 57.7 \\
\hline & $201-350$ & 411 & 32.8 \\
\hline & $>351$ & 119 & 9.5 \\
\hline \multirow[t]{2}{*}{ TB at initiation } & Yes & 94 & 7.5 \\
\hline & No & 1160 & 92.5 \\
\hline \multirow[t]{2}{*}{ Cotrimoxazole } & Yes & 463 & 36.9 \\
\hline & No & 791 & 63.1 \\
\hline \multirow[t]{5}{*}{ ART regimen at initiation } & $\mathrm{AZT} / 3 \mathrm{TC} / \mathrm{EFV}$ & 99 & 7.9 \\
\hline & $\mathrm{AZT} / 3 \mathrm{TC} / \mathrm{NVP}$ & 584 & 46.6 \\
\hline & $\mathrm{TDF} / 3 \mathrm{TC} / \mathrm{EFV}$ & 513 & 40.9 \\
\hline & $\mathrm{TDF} / 3 \mathrm{TC} / \mathrm{NVP}$ & 41 & 3.3 \\
\hline & Others & 17 & 1.4 \\
\hline \multirow{2}{*}{$\begin{array}{l}\text { Self-reported poor } \\
\text { compliance history }\end{array}$} & Yes & 274 & 21.9 \\
\hline & No & 980 & 78.1 \\
\hline
\end{tabular}


nervous (PNS), musculoskeletal (MSK), hematological (HMT), hepatic and renal (HR), and systemic symptoms (SS).

\section{Occurrence of ADRs}

Among the 1,254 patients who initiated treatment at the Day Care Unit of the Yaounde Central Hospital during the study period, a total number of $306(24.40 \%)$ individuals had at least one ART-associated ADR reported; this represented the overall prevalence. Of these, $134(43.8 \%)$ reported at least two ADRs and three of them reported three ADRs. The most common biological systems affected by the first adverse reactions were HMT, systemic, GI, DMT, and the CNS with $37.58 \%, 12.75 \%, 12.75 \%$, $12.75 \%$, and $10.78 \%$, respectively (Table 4 ).

\section{Proportion of ADRs in different systems as per ART regimen}

Table 5 shows that the highest numbers of ADRs were observed with AZT/3TC/NVP (65.3\%), followed by TDF/3TC/ EFV (20.91\%). This cross-tabulation between the ADRs in different biological systems by ART regimen showed that there is an association between the distribution of ADRs according to the initial ART regimen and system affected $(p<0.001)$.

\section{Onset time of ADRs}

Based on clinical records, the onset of ADR for each patient was determined as the time that elapsed between ART initiation and the date of complaint or date reported on test results. Table 6 shows the onset time of ADR, in $88.56 \%$ of individuals who reported ADRs; it had occurred between 0 and 3 months of treatment.

\section{Association between selected variables and ADRs}

Age was a statistically significant independent predictor of ADRs in our study $(p=0.003)$. Individuals older than 44 years of age were more susceptible to develop these conditions compared to the other age groups. Having a relatively low CD4 count of 200 cells $/ \mathrm{mm}^{3}$ or less was significantly associated with occurrences $(p=0.004)$. In the study, it was also found that cotrimoxazole prophylaxis intake and initial ART regimen were associated with the occurrence of $\operatorname{ADR}(p=0.029$ and $p<0.001$, respectively). Sex, weight, and WHO's clinical stage were not significantly associated with the development of ADRs in our study population (Table 7).

\section{DISCUSSION}

The development of ADRs among HIV patients undergoing ARV treatment is one of the most limiting factors that compromise compliance and adherence and is the major cause of treatment changes as shown by many studies. Moreover, ADRs are one of the reasons for loss to follow-up of ART and deaths.

Table 3. General profile of specific ADRs by systems or groups.

\begin{tabular}{|c|c|c|c|c|c|c|c|c|}
\hline System & GI & DMT & CNS & MSK & SS & HMT & HR & PNS \\
\hline \multirow[t]{8}{*}{ Specific ADRs } & -abdominal pains & -rash & -headache & -myalgia & -fatigue & -mild to severe anemia & -increased ALAT/ASAT & -tingling \\
\hline & -diarrhea & -pruritus & -dizziness & -arthralgia & -asthenia & & -increased creatinemia & -numbness \\
\hline & -nausea/ & -blue nails & -insomnia & -parathesia & -dysnoea & & & \\
\hline & -vomiting & -hyper-pigmentation & -drowsiness & & & & & \\
\hline & -anorexia & -Steven Johnson syndrome & -nightmares & & & & & \\
\hline & & & -vision reduction & & & & & \\
\hline & & & -memory loss & & & & & \\
\hline & & & -allucinations & & & & & \\
\hline
\end{tabular}

Table 4. Number of ADRs in different biological systems.

\begin{tabular}{|c|c|c|c|c|c|c|c|}
\hline \multicolumn{8}{|c|}{ Number of patients with ADR by system } \\
\hline GI & DMT & CNS & MSK & SS & HMT & HR & PNS \\
\hline $39(12.75 \%)$ & $39(12.75 \%)$ & $33(10.78 \%)$ & $3(0.98 \%)$ & $39(12.75 \%)$ & $115(37.58 \%)$ & $18(5.88 \%)$ & $20(6.53 \%)$ \\
\hline
\end{tabular}

Table 5. Preferential distribution of the ADRs as per the ART regimen.

\begin{tabular}{|c|c|c|c|c|c|c|c|c|c|c|}
\hline & & \multicolumn{8}{|c|}{ Number of patients with ADR by system } & \multirow{2}{*}{ Total } \\
\hline & & GI & DMT & CNS & MSK & SS & HMT & HR & PNS & \\
\hline \multirow{5}{*}{$\begin{array}{l}\text { ART } \\
\text { regimen }\end{array}$} & Others & 0 & 0 & 0 & 0 & 0 & 1 & 0 & 0 & $1(0.34 \%)$ \\
\hline & $\mathrm{AZT} / 3 \mathrm{TC} / \mathrm{EFV}$ & 3 & 3 & 9 & 0 & 0 & 16 & 0 & 2 & $33(10.78 \%)$ \\
\hline & $\mathrm{AZT} / 3 \mathrm{TC} / \mathrm{NVP}$ & 24 & 27 & 6 & 1 & 18 & 98 & 13 & 12 & $199(65.03 \%)$ \\
\hline & TDF/3TC/EFV & 10 & 7 & 17 & 1 & 19 & 0 & 5 & 5 & $64(20.91 \%)$ \\
\hline & TDF/3TC/NVP & 2 & 2 & 1 & 1 & 2 & 0 & 0 & 1 & $9(2.94 \%)$ \\
\hline Total & & $39(12.75 \%)$ & $39(12.75 \%)$ & $33(10.78 \%)$ & $3(0.98 \%)$ & $39(12.75 \%)$ & $115(37.58 \%)$ & $18(5.88 \%)$ & $20(6.53 \%)$ & $306(100 \%)$ \\
\hline
\end{tabular}

$\mathrm{AZT}=$ Zidovudine; $3 \mathrm{TC}=$ Lamivudine EFV $=$ Efavirenz $; \mathrm{NVP}=$ Nevirapine; $\mathrm{TDF}=$ Tenofovir $p=0.000$. 
Table 6. Onset of ADR from ART initiation.

\begin{tabular}{cc}
\hline Onset of ADRs & $\boldsymbol{n}(\mathbf{\%})$ \\
\hline $0-3$ months & $271(88.56)$ \\
$4-6$ months & $23(7.52)$ \\
$7-12$ months & $8(2.61)$ \\
$>12$ months & $4(1.31)$ \\
\hline
\end{tabular}

Table 7. Logistic regression analysis of selected variables and ADR.

\begin{tabular}{|c|c|c|c|c|}
\hline \multirow{2}{*}{ Variables or categories } & & \multicolumn{2}{|c|}{ ADRs } & \multirow{2}{*}{$p$ value } \\
\hline & & NO & YES & \\
\hline \multirow[t]{2}{*}{ Sex } & $F$ & 643 & 202 & $p=0.556$ \\
\hline & $M$ & 305 & 104 & \\
\hline \multirow[t]{4}{*}{ Age } & $15-24$ & 74 & 16 & $p=0.003^{*}$ \\
\hline & $25-34$ & 341 & 89 & \\
\hline & $35-44$ & 302 & 96 & \\
\hline & $>44$ & 231 & 105 & \\
\hline \multirow[t]{3}{*}{ Weight } & $<50$ & 96 & 26 & $p=0.604$ \\
\hline & $50-60$ & 322 & 111 & \\
\hline & $>60$ & 530 & 169 & \\
\hline \multirow[t]{4}{*}{ WHO stage } & I & 316 & 91 & $p=0.712$ \\
\hline & II & 262 & 89 & \\
\hline & III & 310 & 106 & \\
\hline & IV & 60 & 20 & \\
\hline \multirow[t]{3}{*}{ CD4 count at initiation } & $\leq 200$ & 528 & 196 & $p=0.004$ \\
\hline & $201-350$ & 317 & 94 & \\
\hline & $>350$ & 103 & 16 & \\
\hline \multirow[t]{2}{*}{ Cotrimoxazole prophylaxis } & Yes & 334 & 129 & $p=0.029$ \\
\hline & No & 614 & 177 & \\
\hline \multirow[t]{5}{*}{ Initial ART regimen } & Others & 16 & 1 & $p=0.000$ \\
\hline & $\mathrm{AZT} / 3 \mathrm{TC} / \mathrm{EFV}$ & 66 & 33 & \\
\hline & $\mathrm{AZT} / 3 \mathrm{TC} / \mathrm{NVP}$ & 385 & 199 & \\
\hline & $\mathrm{TDF} / 3 \mathrm{TC} / \mathrm{EFV}$ & 449 & 64 & \\
\hline & $\mathrm{TDF} / 3 \mathrm{TC} / \mathrm{NVP}$ & 32 & 9 & \\
\hline
\end{tabular}

A total of 1,254 HIV/AIDS patients who initiated ART were included in this study, and among them, 306 had developed at least one ADR, resulting in an overall prevalence of $24.5 \%$. Among the 306 individuals, 134 (43.8\%) reported two ADRs and three reported three ADRs. The prevalence reported in this study was lower than that obtained in many African and non-African countries. Several studies in India have reported a very high prevalence of $75.65 \%$ (Bhatnagar et al., 2013), 81.42\% (Kumari et al., 2017), and 90\% (Shet et al., 2014). Such high values were equally found in Iranian and Ethiopian populations with $87.6 \%$ and $89.8 \%$, respectively (Khalili et al., 2009; Tadesse et al., 2014). Some research groups in Ghana, Nigeria, and Ethiopia reported values of $9.4 \%, 4.6 \%, 6.4 \%$, and $4.3 \%$, respectively, which were considerably lower than what was found in the present study population (Eluwa et al., 2012; Kindie et al., 2017; Lartey et al., 2014; Lorío et al.,
2014). Some studies carried out in India and Brazil reported a prevalence very close to what was found in this study, with $21 \%$ and 20.2\%, respectively (Roshni et al., 2016; Santini-Oliveira et al., 2014). A Cameroonian study carried out at the Douala Reference Hospital found the prevalence of ADR to be $19.5 \%$ (Luma et al., 2012), which was also very close to what we found at the Yaounde Central Hospital. There is a possibility that not all patients' complaints were reported as adverse reactions, thus leading to an underestimation of their real prevalence in the study population.

From the study population, it was observed that the HMT system was most frequently affected, with a prevalence of $37.58 \%$ recorded from 115 cases of ADRs that consisted mainly of mild-to-severe anemia. These results were in line with many other studies which also found that anemia was the most frequently reported ADR (Bhatnagar et al., 2013; Lartey et al., 2014; Malalur et al., 2016; Roshni et al., 2016). On the other hand, these results were in contrast with other studies that reported GI disorders as the most frequent ADRs (Khalili et al., 2009; Kumari et al., 2017; Raikar et al., 2018). Yet, other studies regarding the most common side effects reported pains (Eluwa et al., 2012), peripheral neuropathy (Luma et al., 2012), and CN system ADRs (Lorío et al., 2014). It is important to implement intense laboratory monitoring to quickly diagnose drug toxicity, in addition to the clinical diagnosis.

The vast majority of adverse reactions occurred during the first 6 months of treatment; these findings are consistent with some previous studies (Eluwa et al., 2012; Ibrahim Elnagar, 2017; Kindie et al., 2017). An explanation was proffered that early advent of ADRs is host-dependent. It is important to closely monitor patients within this time frame to prevent and avoid failure in compliance. This goes with the need to improve documentation of ADR occurrences as well (Duval et al., 2004).

There was an association between the initial ART regimen and the occurrence of ADRs in general and according to the distribution in different systems $(p<0.001)$. These results are in line with those reported in other studies (Eluwa et al., 2012; Luma et al., 2012; Masenyetse et al., 2015; Shet et al., 2014). EFV was mainly associated with the CNS ADRs, AZT with anemia, NVP with DMT problems and liver toxicity, and TDF with renal toxicity. Similar associations were found elsewhere (Ejigu et al., 2018; Kumari et al., 2017).

In this study, HIV-positive patients older than 45 years of age were more at risk of these reactions than patients in the other age groups. These findings are in line with studies carried out in India (Bhatnagar et al., 2013; Kumari et al., 2017; Masenyetse et al., 2015) where the patient's age was significantly associated with the developed ADRs. They were, however, in contradiction with the results published in Nigeria and India (Eluwa et al., 2012; Malalur et al., 2016; Shet et al., 2014).

CD4+ T-cell count was the other important factor. Patients with CD4 below 200 cells at the beginning of the treatment were more likely to develop ADRs than patients with cell counts higher than that. This result is in agreement with others studies in India (Bhatnagar et al., 2013; Kumari et al., 2017; Shet et al., 2014), suggesting that the initiation of treatment at low CD4 
cell counts could have a negative impact on treatment outcomes. On the contrary, it was found in Ethiopia and Nigeria that CD4 cell count was not associated with the development of ADRs (Eluwa et al., 2012; Kindie et al., 2017).

Cotrimoxazole, which is used in the prevention of opportunistic infections, was found to impact on the development of ADRs. Patients who did not receive it were more susceptible to developing ADRs compared to those who received it. This is in agreement with a study carried out in Ethiopia (Kindie et al., 2017), which revealed that this prophylaxis helps in preventing adverse reactions. The explanation can be that prophylactic therapy successfully lessens the incidence of ADRs and ameliorates the quality of life for people infected. Nevertheless, a study carried out in India revealed that this treatment was not associated with ADRs (Shet et al., 2014).

Sex, weight, and WHO's stage were not statistically associated with the development of ADRs in this study. These results are in line with those reported by Eluwa et al. (2012), Malalur et al. (2016), and Shet et al. (2014), but contradict those conducted in Ethiopia and Ghana (Ejigu et al., 2018; Lartey et al., 2014). Some studies also showed that female HIV patients were more at risk of developing nevirapine-related skin rash and hepatotoxicity (Bersoff-Matcha et al., 2000). Another study showed that $90 \%$ of HIV patients with ART-related ADR were females (Pitt et al., 2009). However, regarding the WHO's clinical stage, it was found in Ethiopia that patients in the advanced stage were more susceptible to develop ADRs (Kindie et al., 2017). This contrariness can be due to possible differences in the study settings, regimen combination, and time gap during which the studies were conducted.

Despite the results obtained, the main limitation of this study was its retrospective nature that may have led to the underestimation of the actual prevalence of ADRs in the facility. Also, some other important predictors that might be associated with study outcome (ADR) are not included.

\section{CONCLUSION}

The spectrum of adverse events in this study was wide and varied, with an overall prevalence of $24.40 \%$. Important information on the prevalence of antiretroviral ADRs and their associated factors has been given for treatment guideline review, pharmaceutical planning, and decision-making. It is important to implement a pharmacovigilance system, which assesses and monitors the safety profiles and the impact of antiretroviral medicines in Cameroon. More research is needed to be carried out to help in developing algorithms for the prediction of adverse effects of the existing regimens. The management of adverse reactions may be done by dose adjustment and the choice of an appropriate regimen. These are key strategies to improve compliance for patients starting ART and to subsequently reduce the patient suffering and improve their quality of life.

\section{ACKNOWLEDGMENTS}

The authors acknowledge the contribution of the staff of the Yaounde Central Hospital.

\section{CONFLICT OF INTEREST}

All authors declared that they have no conflicts of interest.

\section{FUNDING}

None.

\section{AUTHORS' CONTRIBUTIONS}

All authors contributed equally to the work.

\section{REFERENCES}

Anlay DZ, Alemayehu ZA, Dachew BA. Rate of initial highly active anti-retroviral therapy regimen change and its predictors among adult HIV patients at University of Gondar Referral Hospital, Northwest Ethiopia: a retrospective follow up study. AIDS Res Ther, 2016; 13:10; doi. org/10.1186/s12981-016-0095-x

Bersoff-Matcha SJ, Miller WC, Aberg JA, Powderly WG, Mundy LM. Sex differences in Nevirapine rash. Clin Infect Dis, 2000; 32:124-9.

Bhatnagar S, Sharma H, Sharma VK. Study of adverse effects of Anti-Retroviral Therapy in HIV naive patients and their association with CD4 cell count. Asian J Pharm Clin Res, 2013; 6:122-3.

Cohen MS, Chen YQ, McCauley M, Gamble T, Hosseinipour MC, Kumarasamy N, Hakim JG, Kumwenda J, Grinsztejn B, Pilotto JHS, Godbole SV, Chariyalertsak S, Santos BR, Mayer KH, Hoffman IF, EshlemanSH, Piwowar-Manning E, Cottle L, Zhang XC, Makhema J, Mill LA, Panchia R, Faesen S, Eron J, Gallant J, Havlir D, Swindells S, Elharrar V, Burns D, Taha TE, Nielsen-Saines K, Celentano DD, Essex M, Hudelson SE, Redd AD, Fleming TR. Anti-retroviral therapy for the prevention of HIV-1 transmission. N Engl J Med, 2016; 375:830-9.

Duval X, Journot V, Leport C, Chene G, Dupon M, Cuzin L, May T, Morlat P, Waldner A, Salamon R, Raffi F, Antiprotease Cohort (APROCO) Study Group. Incidence of and risk factors for adverse drug reactions in a prospective cohort of $\mathrm{HIV}$-infected adults initiating protease inhibitor-containing therapy. Clin Infect Dis, 2004; 39:248-55.

Ejigu A, Gehzu M, Haileselassie W. Adverse drug reactions causing treatment change among patients taking highly active anti-retroviral therapy in health care facilities of Mekelle, Ethiopia. J Appl Pharm Sci, 2018; 8:104-10.

Eluwa GI, Badru T, Akpoigbe KJ. Adverse drug reactions to anti-retroviral therapy (ARVs): incidence, type and risk factors in Nigeria. BMC Clin Pharmacol, 2012; 12:7; doi:10.1186/1472-6904-12-7

Fagbami O, Oluwasanjo A, Fitzpatrick C, Fairchild R, Shin A, Donato A. Factors supporting and inhibiting adherence to HIV medication regimen in women: a qualitative analysis of patient interviews. Open AIDS J, 2015; 9:45-50.

Hansana V, Sanchaisuriya P, Durham J, Sychareun V, Chaleunvong K, Boonyaleepun S, Schelp FP. Adherence to anti-retroviral therapy (ART) among people living with HIV (PLHIV): a cross-sectional survey to measure in Lao PDR. BMC Public Health, 2013; 13:617; doi:10.1186/1471-2458-13-617.

Hawkins T. Understanding and managing the adverse effects of anti-retroviral therapy. Antiviral Res, 2010; 85:201-9.

Ibrahim Elnagar BM. Prevalence and extent of adverse drug reactions in Sudanese patients in highly active anti-retroviral therapy regimens. Int J Pharmacovigil, 2017; 2:1-12.

Khalili H, Dashti-Khavidaki S, Mohraz M, EtghaniA, Almasi F. Antiretroviral-induced adverse drug reactions in iranian human immunodeficiency virus-positive patients. Pharmacoepidemiol Drug Saf, $2009 ; 18: 848-57$

Kindie E, Alamrew AZ, Worku E. Time to development of adverse drug reactions and associated factors among adult HIV-positive patients on antiretroviral treatment in Bahir Dar City, Northwest Ethiopia. PLoS One, 2017; 12(12):e0189322. 
Kumari R, Chandra S, Gari M, Kumari A. An assessment of adverse drug reaction patterns among HIV-positive patients receiving anti-retroviral therapy in a tertiary care hospital. Int J Pharmacol R, 2017; 07:88-93.

Lartey M, Asante-Quashie A, Essel A, Kenu E, Ganu V, Neequaye A. Adverse drug reactions to antiretroviral therapy during the early art period at a tertiary hospital in Ghana. Pan Afr Med J, 2014; 18:25; doi:10.11604/pamj.2014.18.25.3886

Lima DGL, Gomes de Arruda EA, Alves de Lima AJ, Oliveira E, França FMM. Factors determining changes in initial antiretroviral therapy. Rev Assoc Med Bras, 2012; 58:222-8.

Lorío M, Colasanti J, Moreira S, Gutierrez G, Quant C. Adverse drug reactions to antiretroviral therapy in HIV-infected patients at the largest public hospital in Nicaragua. J Int Assoc Provid AIDS Care, 2014; 13:466-70.

Luma HN, Doualla MS, Choukem SP, Temfack E, Ashuntantang G, Achu H, Koulla-Shiro S. Adverse drug reactions of Highly Active Antiretroviral Therapy (HAART) in HIV infected patients at the General Hospital, Douala, Cameroon: a cross sectional study. AIDS Res Ther, 2012; 9:1-7; Available via http://www.aidsrestherapy.com/content/9/1/35.

Malalur C, Shenoy S, Varma M, Kamath A, Manohar H, Saravu K. Evaluation of adverse drug reaction profile of antiretrovirals in HIVpositive patients in a tertiary care hospital. Natl J Physiol Pharm Pharmacol, 2016; 6(5):488-93; doi:10.5455/njppp.2016.6.0514520072016.

Masenyetse LJ, Manda S, Mwambi HG. An assessment of adverse drug reactions among HIV positive patients receiving antiretroviral treatment in South Africa. AIDS Res Ther, 2015; 12:6; doi:10.1186/s12981015-0044-0

Mehari M, Kiros N, Yemane A, Asghedom N, Debesay S, Tekeste T. Factors affecting treatment adherence among HIV-positive patients in Eritrea. Int Biol Biomed J, 2017; 3:150-6.

Neuman MG, Schneider M, Nanau RM, Parry C. HIVantiretroviral therapy induced liver, gastrointestinal, and pancreatic injury. Int J Hepatol, 2012; 2012:1-23.

Oumar AA, Diallo K, Dembélé JP, Samaké L, Sidibé I, Togo B, Sylla M, Tounkara A, Dao S, Tulkens PM. Adverse drug reactions to antiretroviral therapy: prospective study in children in Sikasso (Mali). J Pediatr Pharmacol Ther, 2012; 17:382-8.

Pitt J, Myer L, Wood R. Quality of life and the impact of drug toxicities in a South African community-based antiretroviral programme. J Int AIDS Soc, 2009; 12:5; doi:10.1186/1758-2652-12-5

Raikar SR, Patil SB, Rao YV, Raghuveer B. An assessment of adverse reactions to antiretroviral therapy in a South Indian government hospital. Natl J Physiol Pharm Pharmacol, 2018; 8:1405-8.
Rajesh R, Sonika S, Sudha V, Varma DM. Association between medication adherence outcomes and adverse drug reactions to highly active antiretroviral therapy in indian human immunodeficiency virus-positive patients. J Young Pharm, 2012; 4:250-60.

Reust CE. Common adverse effects of antiretroviral therapy for HIV disease. Am Fam J, 2011; 83:1443-51.

Roshni P, Thampi A, Ashok BA, Joy J, Thomas T, Kumar KG. A study on adverse drug reactions of anti-retroviral therapy in HIV patients. Int J Pharm Sci Rev Res, 2016; 36:8-11.

Santini-Oliveira M, Friedman RK, Veloso VG, Cunha CB, Pilotto JH, Marins LMS, João EC, Torres TS, Grinsztejn B. Incidence of antiretroviral adverse drug reactions in pregnant women in two referral centers for HIV prevention of mother-to-child-transmission care and research in Rio de Janeiro, Brazil. Braz J Infect Dis, 2014; 18:372-8.

Shet A, Antony J, Arumugam K, Kumar DS, Rodrigues R, DeCosta A. Influence of adverse drug reactions on treatment success: prospective cohort analysis of HIV-infected individuals initiating first-line antiretroviral therapy in India. PLoS One, 2014; 9(3):e91028; doi:10.1371/ journal.pone.0091028

Shubber Z, Calmy A, Andrieux-Meyer I, Vitoria M, RenaudThéry F, Shaffer N, Hargreaves S, Mills EJ, Ford N. Adverse events associated with nevirapine and efavirenz-based first-line antiretroviral therapy: a systematic review and meta-analysis. AIDS, 2013; 27:1403-12.

Tadesse WT, Mekonnen AB, Tesfaye WH, Tadesse YT. Selfreported adverse drug reactions and their influence on highly active antiretroviral therapy in HIV infected patients: a cross sectional study. BMC Pharmacol Toxicol, 2014; 15:32; doi:10.1186/2050-6511-15-32

UNAIDS/WHO. Report on the global AIDS epidemic. 2019. UNAIDS/WHO/UNICEF. Global AIDS monitoring. 2017.

\section{How to cite this article:}

Nkenfou-Tchinda CN, Tiedeu BA, Nkenfou CN, Nji AM, Mbange AE, Chedjou JPK, Fomboh CT, Kouanfack C, Mbacham WF. Adverse drug reactions and associated factors among adult HIV-positive patients taking art at the Yaounde Central Hospital, Cameroon. J Appl Pharm Sci, 2020; 10(11):067-073. 